\title{
Architecture for animation of affective behaviors in pedagogical agents
}

\author{
Ronaldo Motola ${ }^{*}$, Patrícia Augustin Jaques ${ }^{1}$, Margarete Axt $^{2}$, Rosa Vicari ${ }^{2}$ \\ ${ }^{1}$ Programa de Pós-Graduação em Computação Aplicada- PIPCA, Universidade do Vale do Rio dos Sinos - UNISINOS, \\ Av. Unisinos, 950 - Cristo Rei - 93022-000, São Leopoldo, RS, Brazil \\ ${ }^{2}$ Pós-Graduação em Informática na Educação - PGIE, Universidade Federal do Rio Grande do Sul - UFRGS, \\ Av. Paulo Gama, 110, 90040-060, Porto Alegre, RS, Brazil
}

A previous version of this paper appeared at SBIE 2009

(XX Brazilian Symposium on Informatics in Education)

Received: March 31, 2009; Accepted: July 30, 2009

\begin{abstract}
This article introduces an open-source module responsible for the presentation of verbal (speech) and corporal (animation) behaviors of animated pedagogical agents. This module can be inserted into any learning environment regardless of application domain and platform, being executable under different operating systems. It was implemented in Java as a reactive agent (named Body agent) that communicates with the agent's Mind through a language known as FIPA-ACL. Therefore, it may be inserted into any intelligent learning environment that is also capable to communicate using FIPA-ACL. Persistence of information is ensured by XML files, increasing the agent's portability. The agent also includes a mechanism for automatically updating new behaviors and characters once available in the server. A simulation environment was conceived to test the proposed agent.
\end{abstract}

Keywords: animated pedagogical agents, emotions, affective computing, lifelike agents.

\section{Introduction}

Due to the motivational aspect of lifelike characters, the interfaces of computational systems are being enriched with characters that exhibit body and facial expressions. These human characteristics, coupled with a good user interface dialogue, turn systems more attractive because they explore more natural user interaction modes. Some applications in education, for instance, employ animated agents to present students with pedagogical content along with demonstrations aimed at engaging and motivating them ${ }^{1-3}$. These agents are known as Animated Pedagogical Agents (APAs).

APAs are intelligent agents that play a pedagogical or educational role in order to facilitate or improve learning, being personified by lifelike characters who interact with students. The agents employ multimedia resources to display an animated character with characteristics similar to those of intelligent beings. Thus, unlike conventional systems, the APAs communication assumes a more anthropomorphic and social nature. They exploit the natural tendency of people to engage in social interactions with computers termed as The Media Equation ${ }^{4}$. Some examples of APAs include: Adele ${ }^{5,6}$, Steve $^{7}$, Vincent ${ }^{8}$, Cosmo $^{1}$ and others ${ }^{9}$. Using APAs for educational purposes brings forth new possibilities for computing learning systems as they can, for example, demonstrate tasks ${ }^{10}$, use locomotion and gestures to focus student attention on the most important aspects of a task ${ }^{11}$, and respond emotionally to a student ${ }^{3,12,13}$. APAs offer a great promise by enhancing the communication capacity of educational systems ${ }^{5}$ and their ability to engage and motivate students ${ }^{14}$.

The pedagogical tactics applied in learning environments may be of two types: affective tactics or tactics for performance and competence ${ }^{\mathrm{I}}$. The latter consist of actions that support students in learning domain concepts ${ }^{15}$. The affective tactics, on the other hand, aim to adapt the system to the affective state of the student, promoting a positive mood and providing emotional support. Such tactics are typically represented by emotive corporal and facial animations accompanied by encouraging speeches, which we call affective behaviors.

The architecture of an APA capable of applying affective tactics is generally composed by two main modules ${ }^{2,16}$ : the (i) Mind, responsible for updating the student's model and choosing an appropriate affective tactic to apply; and the (ii) Body, which exhibits the agent's verbal (speeches) and behavioral (animations) actions that represent the chosen tactic. Although the agent's Mind should be designed specifically for the educational environment inhabited by the lifelike agent, since the choice of a suitable tactics must consider the student's profile, the educational subject and the pedagogical theory that grounds the system, the functionalities of

I. This separation has been adopted by the Group of Artificial Intelligence at the Universidade do Rio Grande do Sul. Other synonyms for "tactics for performance and competence" are "domain-based tactics" and "cognitive tactics". 
the Body are typically the same in all educational systems, particularly for Web-based systems. However, a new implementation is usually required in each case, as systems rely on platform dependent technologies or agents are designed to work in specific environments.

We propose a solution focused on the presentation of behaviors that seek to promote certain emotions on the students rather than exhibiting affective behaviors that represent emotions the agent itself is "feeling". This paper describes the architecture and implementation of an opensource, domain and platform independent Body module for 2D APAs, that has been conceived to be reused in multiple learning environments. To ensure domain and application independence (implying it may be embedded in applications developed for different subjects) and also platform independence (it is suitable for educational environments running in different operating systems), this module (called Body agent) was implemented as a reactive agent in Java ${ }^{18}$ that communicates with the Mind module through the FIPA-ACL ${ }^{19}$ agent communication language. The agent may therefore be inserted into any learning system capable of communicating using FIPA-ACL.

This article is organized as follows: Section 2 presents the educational advantages of adopting APAs in learning environments as observed by researchers. Section 3 introduces the area of Affective Computing, mainly focusing on the Expression of Emotions. Section 4 describes some related works, comparing them with the proposed agent. In Section 5, the architecture and implementation aspects of the proposed agent are detailed. Section 6 lists the characters available in its current version, and Section 7 describes its mechanisms of believability. Section 8 presents the component of automatic updating of new versions of the agent in the server. Section 9 explains how the Body Agent is integrated with learning systems. Finally, Section 10 delineates some final considerations.

\section{Animated Pedagogical Agents and their Educational Benefits}

APAs adopt a varied repertoire of emotive behaviors to respond to students with facial expressions and emotional gestures and can also employ the audio and visual channels for more effective communication. Thus, they have some advantages over other educational systems or traditional intelligent tutors, as pointed out by Johnson and colleagues $^{20}$.

An APA can demonstrate a task, which may be more effective than describing it. Moreover, an interactive demonstration by an agent offers advantages over a recorded video, as the student has more freedom to move within the virtual world and perceive all sorts of demonstrations from different perspectives. He can even interrupt the demonstration with questions or request the agent itself to finish the task.

The agents can also guide students inside the learning environment. Employing them as navigational guides is an important instructional resource in 3D Worlds, as students often become disoriented in 3D immersive environments ${ }^{20}$.

An APA may also focus a student's attention on certain aspects of the task through gesture and gaze. This can be done in several ways, such as pointing to objects, looking at objects being manipulated by the student, facing the student while waiting for an action, or speaking to him.

An additional property of APAs is the non-verbal communication. For example, the Body agent includes some behaviors (applaud, tap down, and others) that demonstrate approval, disapproval and waiting. The ability to present non-verbal behavior enables a wider variety of feedback levels than those available in traditional tutorial systems. Non-verbal behaviors, such as facial expressions, can be preferable because they may be perceived as less impertinent than a verbal commentary. For instance, a head movement can calm down a student without interrupting him.

Agents also can employ non-verbal conversation signals to emphasize certain aspects of their speeches. For example, an agent may frown, blink the eyes, or move its head to highlight words or sentences, resources that enable a more familiar face-to-face communication dialogue with users.

Although usually employed as individual tutors, APAs may also play the role of study friends or partners in collaborative activities. In many situations, group tasks play an important role and students must coordinate their actions with those of their colleagues. In this scenario, a pedagogical agent can work as a virtual friend, assisting students in group activities when colleagues are not available.

Recent studies demonstrated that animated agents that display a computational model of emotion can be more effective pedagogically. They provide a strong motivational effect on students and emotion plays an important role in motivation. This motivational aspect was observed even in agents that did not display a sufficiently pro-active behavior ${ }^{14}$. An evaluation with the Cosmo agent ${ }^{14}$ showed that even a mute agent, which does not offer any type of support to students, has a motivational effect over them.

Johnson et al. ${ }^{20}$ state that in addition to the aforementioned benefits stemming from the character's presence, an APA must also have the same pedagogical abilities of an intelligent tutoring system. In order to be helpful it should know how to answer a student's question, generate explanations, ask questions to students and infer student's ability levels.

\section{Affective Computing}

The important role of affect in learning motivates researchers in the field of Computer in Education to investigate how to employ Artificial Intelligence techniques to enrich educational systems with the ability of inferring and expressing emotions. This research topic is called "Affective Computing" (AC), defined by Picard ${ }^{21}$ as "computing that relates to, arises from or deliberately influences emotions". This field is split into two major research branches. The first one studies mechanisms to recognize human emotions or to express emotions by machine in human-computer interaction. 
The second branch investigates how to simulate emotion in machines (emotion synthesis) in order to find out more about human emotions and to construct more realistic robots.

AC research applied to Education falls generally in the first branch, as it mainly investigates mechanisms to recognize student's emotions and also to express emotions in the interaction between an artificial tutor and a student.

A system must interpret the student's emotions correctly in order to adapt itself to the student's affect. For example, a student disappointed with his performance will probably abandon a task. The system should identify the situation in order to encourage him to carry on. As such, it must include both a cognitive model and an emotional model of the student that register his affective history: all the emotions he felt while interacting with the educational system. This affective model must be dynamic enough to account for changes in emotional states.

Actually, we identify four major modes of user emotion recognition: (1) voice (prosody); (2) observable behavior, i.e. user's actions in the system interface (for example, chosen options and typing speed); (3) facial expressions; and (4) physiological signs (blood volume pulse, electromyogram muscle tension, skin conductivity, breathing).

Picard's group achieved good results in the recognition of eight emotional states (neutral, anger, hate, grief, platonic love, romantic love, joy and reverence) by assessing physiological signs, with a success rate of about $81 \%$. These results were obtained from tests conducted with one person in the course of twenty days, spanning about 5 weeks. The signals measured were: blood volume pulse, electromyogram (muscle tension), skin conductivity, and breathing. Kaiser and Wehrle ${ }^{23}$ worked on video-based facial expression recognition of emotion.

The user's observable behavior is still one of the most natural interaction modes between user and computer. Martinho and colleagues ${ }^{24}$ state that in a well-defined context (an educational environment), it may be a path to predict, recognize and interpret user's affective states, an approach denominated Cognitive-Based Affective User Modeling $(\mathrm{CB}-\mathrm{AUM})^{24}$.

An educational system may express emotions just as empathic teachers do in a presence class. When capable of displaying emotions they can motivate and engage students in learning, amuse and promote positive emotions in them, which is an upswing for a more effective learning ${ }^{17,25}$. In order to convey emotions educational systems are generally implemented as APAs. Agents designed to interact affectively with students show emotive behaviors that consist of animations of a lifelike character.

The next section describes some techniques employed by $\mathrm{AC}$ to express emotions in the machine.

\subsection{The expression of emotions in machines}

Because they are represented by lifelike characters, APAs become a powerful tool to express emotions in a machine through face and body signals.
In order to express emotions lifelike pedagogical agents show animations composed by emotive facial expressions and body attitudes, and affective utterances. The agents choose their affective behavior from a library of physical (agent's animations) and verbal behaviors (speeches), alternatively behaviors may be generated dynamically with 3D graphical algorithms.

In the first case, the character's animated behaviors are stored (generally as audio and picture files) in a database of speeches and attitudes, from which the agent chooses a behavior to display. This type of implementation was denominated "Space of Behaviors" by Lester et al. ${ }^{26}$ and was employed to generate animated behaviors for the Cosmo agent (see Section 4 for more details about Cosmo). In order for the agent to retrieve the movements correctly they must be stored with some associated affective information. In Cosmo, for instance, all behaviors in the Space of Behaviors are individually mapped to the emotive state they express.

The alternative implementation is to generate the agent's behavior dynamically with 3D graphical algorithms, a substantially complex task. This kind of implementation is generally adopted for agents inserted into 3D environments.

\section{Related Works}

Cosmo is an agent that inhabits the Internet Advisor, a learning environment for the domain of Internet packet routing. Its function is to demonstrate and to advise students, in real time, on the best way to ship packets for one definitive destination, in a virtual world of routers ${ }^{1}$. Cosmo resembles a curious being with antennas and it is very similar to a small humanoid robot. It can carry out a large variety of behaviors, such as moving, pointing, blinking the eyes, inclining, clapping, and raising and folding its antennas. As for verbal behavior, it has 240 elocutions that vary between 1-20 seconds. One of its architectural components is the Emotivekinesthetic Behavior Sequencing Engine ${ }^{27}$, responsible for mounting and selecting the visual attitudes to be displayed in specific situations. This module was based on the Affective Reasoner framework ${ }^{28}$, which associates emotional states to communication. Cosmo has a repertoire of corporal emotive behaviors associated to speech acts in accordance to its intention or kinesthetic expression. When the Explanation System is invoked to construct a communication plan, it examines the problem state, an information net about the course and the student's model to determine the pedagogical speech action to be employed to communicate with the student. These speech actions are sent to the Emotive-kinesthetic Behavior Sequencing Engine that selects from all emotive behaviors one that expresses the appropriate affective state of the speech act. This is possible because all behaviors from the Space of Behaviors are mapped into the emotive states they express. The main difference between Cosmos's Emotivekinesthetic Behavior Sequencing Engine and the proposed Body Agent is that the former is totally platform-dependent and can only rely on a predefined number of verbal behaviors since the elocutions are recorded. 
Early works to address the presentation of behaviors of an APA on a Web-based learning system were ${ }^{11,29}$. The authors created a script language in order to determine the behavior sequence of the agent. Although it allows determining the behavior of different agents in any Web application, the language does not consider the presentation of emotional behaviors.

Other contributions proposed proprietary architectures for APAs capable of expressing emotions ${ }^{12,30}$. In addition to being dependent of the proposed agent, they were conceived to express the emotions felt by the agent rather than emotions aimed at fostering learning.

Several architectures have been proposed recently ${ }^{12,31-33}$ for 3D embodied agents capable of showing emotional behaviors. These solutions include an architecture for emotion synthesis responsible for evaluating the environment situations and labeling the agent's state as an emotion. Again, the module responsible for presenting emotive behaviors focuses on selecting those actions that convey agent's emotions and not the affective state the agent intends to promote on the student. It is important to consider that virtual agents can experience emotions, for example, becoming indignant with a student, which is hardly the most appropriate reaction to encourage learning. Those architectures have been created for 3D agents, generally conceived to inhabit 3D worlds.

\section{Agent's Description}

This work considers the Body and Mind module of an intelligent and lifelike pedagogical agent as autonomous agents that communicate between themselves. The Mind agent is an intelligent cognitive agent responsible for the affective and performance diagnostic of the student, as well as to determine the best pedagogical tactic to be applied. On the other hand, the Body module is a reactive agent that shows a physical and verbal behavior that best represents the tactic chosen by the Mind Agent. For example, if the Mind chooses the tactic "increase student self-ability" (employed by the agent $\left.\mathrm{Pat}^{34}\right)$, aimed at improving the student's own judgment of what he can do with his skills, the Body should choose a verbal and a physical behavior in order to apply this tactic.

The tactics already inserted in the Body agent database result from previous work on affective pedagogical agents by the authors of this paper (the agent Pat ${ }^{16,35,36}$ ) and were conceived based on pedagogical and psychological studies concerning student's motivation ${ }^{37-39}$ and self-efficacy ${ }^{40}$. They are not detailed here, as only the Mind agent is concerned with deciding which affective tactic to apply, and these tactics and corresponding affective behavior should be designed by its developers. Nevertheless, Section 5.6 describes a scenario demonstrating the interaction between Pat'S Mind and Body agents.

In this section, we describe the architecture of the developed agent that allows it to choose different behaviors for the same tactics in order for the agent to become believable.

\subsection{Agent's architecture}

The architecture of the Body agent, shown in Figure 1, consists of three main modules: (i) a communication module, which handles FIPA-ACL messages sent and received by the agent; (ii) a behavior manager, which chooses a verbal and physical behavior to be displayed according to the tactic to be applied, and (iii) the animation generator, responsible for generating the animation of the selected physical behavior.

The agent works as follows: the Body agent receives a message from the Mind agent containing the tactic to be applied. The Body agent's communication module decodes the message. If it contains a tactic to be applied, it is sent to the Behavior Manager module that verifies in the database which behaviors compose this tactic and chooses an animation and speech of that type to be exhibited. If multiple behaviors are associated to the tactic, the agent will arbitrarily choose one that has not been applied recently. In this case, the agent's behaviors database also stores information on the date and time each behavior was last shown. The behavior and speech identifications are sent to the Animation Generator that generates a dynamic animation from its composing image files. This component consists of Java classes developed by Wilges et al. ${ }^{41}$. The Behavior Manager also sets forth the voice synthesizer for speech.

The image files are stored in a repository called Images, organized in several directories. The directory "MARIA", for example, represents the respective character and the sub-directories "Applaud", "Walk" and "Call" store the files that compose each one of these physical behaviors of the Maria character. In directory "Applaud", the files are named

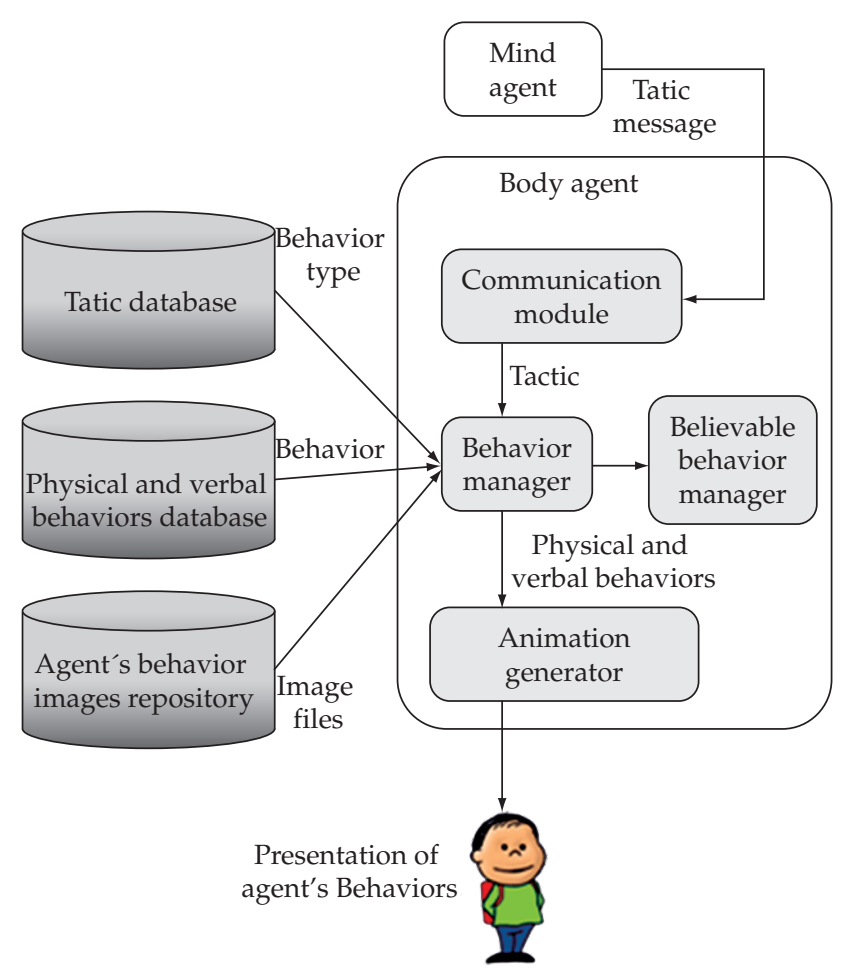

Figure 1. Body Agent's Architecture. 


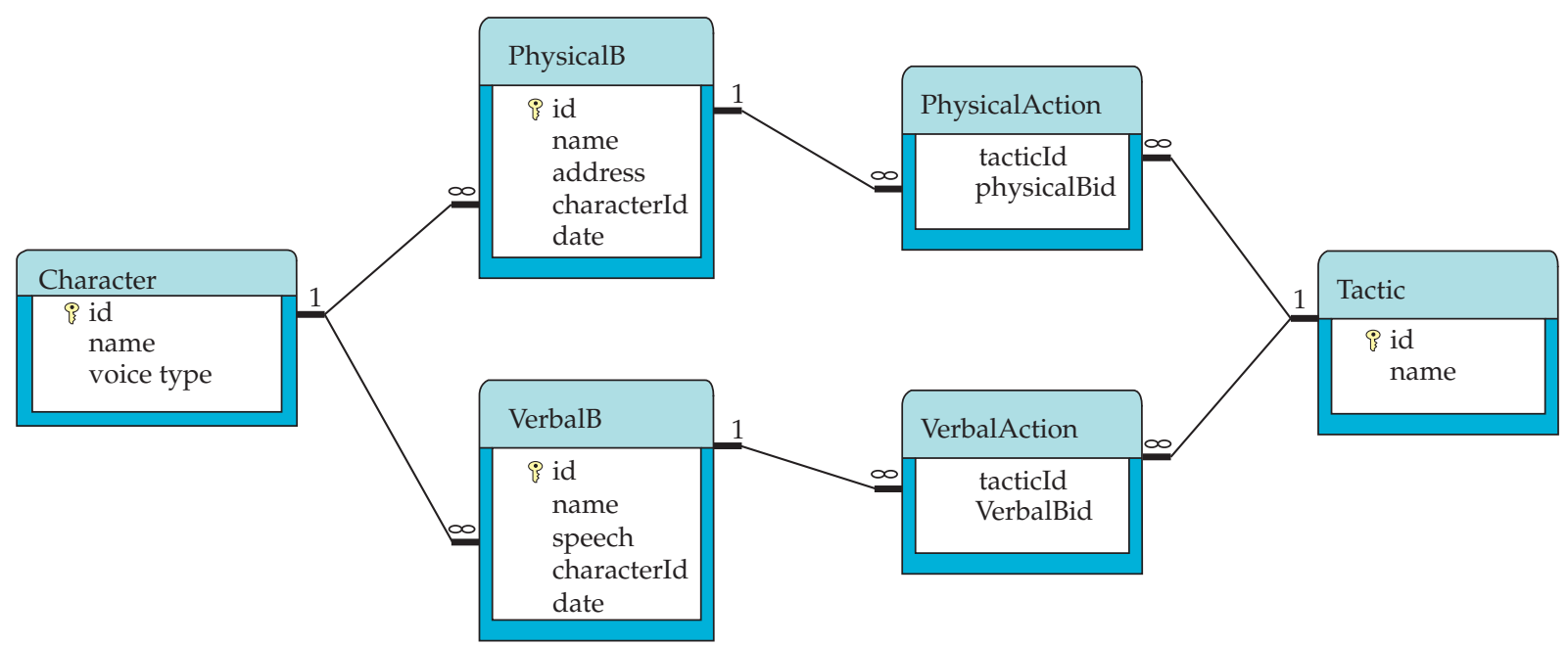

Figure 2. Body agent's Databases Entity-Relationship Model.

applaud00, applaud01, up to applaud10, so that the agent knows the proper image composition sequence to generate the animation.

All the Body agent modules were implemented in Java. FreeTTS v1.2 was employed, which is a free voice synthesizer that adheres to the Java Speech API specification. The agent may be inserted into Web-based learning environments as a Java applet, as well as in stand-alone applications as a separate frame.

\subsection{Description of the agent's databases}

As previously mentioned, the Mind chooses a pedagogical tactic to be applied and sends this information to the Body agent, which should choose a related (verbal and physical) behavior. For the agent to be believable, i.e., to generate the illusion of life and then permit the suspension of disbelief ${ }^{42}$, it should exhibit different animations and speeches for a single situation. Otherwise, the agent's may display mechanical and repetitive behaviors that will cause it to be perceived as predictable and boring by students. The databases were modeled considering the issue of believability.

Figure 2 represents the entity-relationship model ${ }^{43}$ of the three Body agent's databases: (i) a tactics database, (ii) a behaviors database, and (iii) a characters database. The tactics database (named Tactic in Figure 1) stores all possible tactics. The behaviors database (called PhysicalB and VerbalB) stores all physical and verbal behaviors of the agent. A physical behavior is a set of 2D pictures in GIF format. In fact, the database informs the location of the directory that contains this set of pictures. Verbal behaviors are sentences stored in the database to be spoken by the voice synthesizer. Using a voice synthesizer allows the number of verbal behaviors to be easily increased by adding the sentences in natural language into the database. The physical behaviors, although must be designed by a professional designer, can also be easily added by a specific system interface. Two intermediary databases (PhysicalAction and VerbalAction) were added that repre- sent the relation between the agents tactics and behaviors. This intermediary level allows different tactics to be represented by the same behavior and also associating different behaviors to the same tactic. Finally, the characters database (named Character) includes a list of all available agents so that students may choose their preferred character. This database also stores the desired voice timbre for the character from: child-female, young-female, adult-female, aged-female, child-male, young-male, adult-male, and aged-male.

As illustrated in Figure 1 the database Character has a relation one-to-many with the databases PhysicalB and VerbalB, which store physical and verbal behaviors respectively. This is because one character can have many physical and verbal behaviors. These tables also have a relation one-to-many with the databases PhysicalAction and VerbalAction, which link a (verbal or physical) behavior with multiple tactics and vice-versa.

\subsection{Database information persistence in XML}

We adopt XML (eXtensible Markup Language) files, rather than a Database Management System such as MySQL" or Oracle ${ }^{I I I}$ for persistence of information. XML is a language specification that allows programmers to develop their own markup languages to communicate or store information. Using XML files improves system performance and simplifies its installation, and also allows installing the Body agent in embedded systems such as mobile phones.

For persistence of information in XML we apply the framework JDOM ${ }^{\mathrm{IV}}$, which enables reading, manipulating and writing XML files in Java programs. It creates a memory representation of the structure of a XML file, and can also convert Java objects into information stored in the XML format.

In the proposed system, all information manipulated by the agent as tactics, behaviors and characters are converted

II. http://www.mysql.com/

III. http:/ / www.oracle.com/database/index.html

IV. http://www.jdom.org/index.html 
into an XML format. Information from each database is stored in a specific file named as the corresponding database (see Section 5.2). In Figure 3 one observes the XML code relative to the Characters database.

Each XML file has a corresponding Document Type Definition (DTD) file. A DTD file holds information on the structure of an XML document that allows verifying if the XML file adheres to the defined structure. Figure 4 displays the content of file "Characters.dtd" associated to "Characters.xml".

\subsection{Database Information Manager}

The Body agent is entirely configurable through a quite intuitive configuration management interface. A user can add new tactics, behavior types, animations, speeches, characters and even configure time intervals for showing believable behaviors. Figure 5 illustrates the interface for registering verbal behaviors.

\subsection{Communication with other agents}

Communication between agents is an important property in a society of agents that need to exchange information and act together. Information exchange must follow a defined standard to ensure proper successful communication.

The Body agent communicates with the Mind agent using the standard FIPA's Agent Communication Language

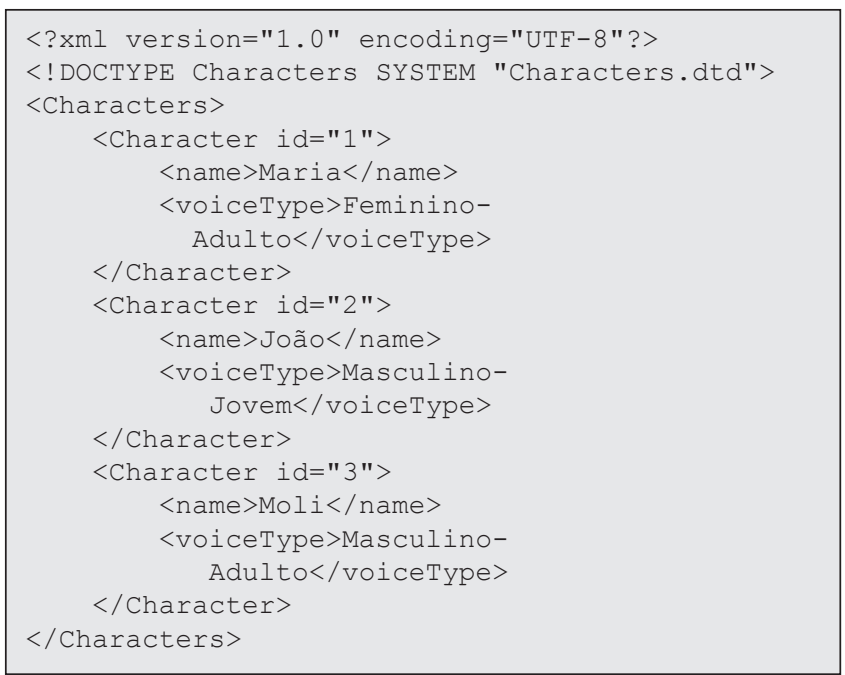

Figure 3. "Characters.xml" file.

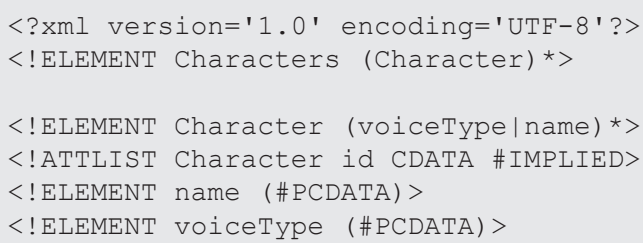

Figure 4. "Characters.dtd" file.
$(A C L)^{V}$. ACL is a proper choice because it is already defined and formalized, thus enabling code reutilization and allowing exchanging messages with or without formatted content. The FIPA-OS framework was adopted because it was developed in Java and has all the resources established by FIPA, including threads to handle communication in FIPA-ACL. The messages received by the agent should have the following FIPA-established format:

- Performative $=$ this field contains a communicative act that represents the agent's desire relative to the information sent in the message. The performative "request" represents a sender request for the receiver to execute some action.

- SenderID = identification of the sender (generally, the Mind agent);

- ReceiverID = receiver identification (Body agent);

- Content $=$ message content. When the message is a "request" (performative), this field contains the tactic to be applied;

- Language $=$ a language that is understood by both the sender and receiver agents. A simple language was created for the proposed system called BodyAgent;

- Protocol $=$ a pattern of sequence messages exchanged by the agents. When the Mind agent requests the Body agent to apply a tactic, the FIPA request protocol is utilized. Figure 6 depicts an example of a message handled by the Body agent.

The exampleillustrates a message, with identifier Mind@ localap, received from the Mind agent. This message tells the Body agent to apply the "IncreaseStudentSelfEfficacy" tactic. The Body agent sends the message to the Behavior Manager, which will display the corresponding behaviors.

V. http://www.fipa.org/

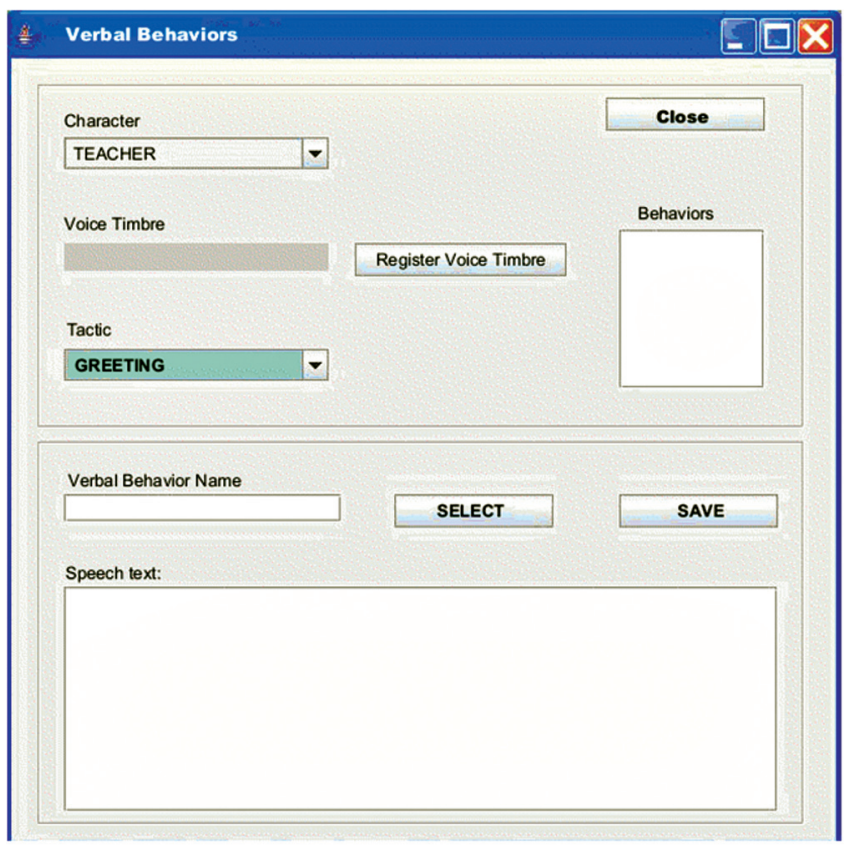

Figure 5. Example of the interface for verbal behaviors registration. 


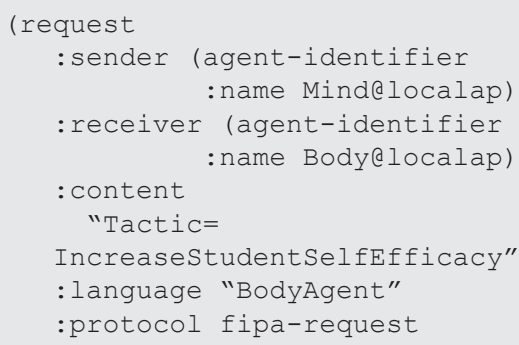

Figure 6. Example of message handled by the Body agent.

\subsection{Fostering emotions in students}

As explained in Section 4, in addition to platform independence, another advantage of the proposed architecture is that it supports agent behaviors aimed at promoting certain emotions in the student rather than exhibiting affective behaviors representing emotions "felt" by the agent itself. Some pedagogical studies show that students who receive suitable emotional support from teachers learn better ${ }^{17}$. Through careful support, teachers can increase student's motivation and confidence. Whereas induced positive emotions appear to foster learning, negative emotions, on the other hand, seem to impair $i^{25}$. Thus, for example, in a situation where a very anxious student faces difficulties to accomplish a set of proposed exercises an agent that reacts with an angry or impatient behavior is unlikely to be helpful.

We believe emotional support may be expressed by tactics presented as animated behaviors and encouragement messages from an APA. These affective tactics are even more effective if they take into account student's emotional states. In this case, the agent should recognize student emotions in order to select the most appropriate affective tactic, as in the case of the Pat agent ${ }^{35,36}$. Therefore, the Mind agent should include mechanisms to infer student emotions and to choose affective tactics accordingly. This is not a function of the Body agent, which can only show affective behaviors that are associated with pre-registered tactics.

In order to better understand the process of selecting an affective tactic and its corresponding behaviors, let us consider an illustrative scenario of the agent Pat as described in Jaques et al. ${ }^{35}$.

Pat's Mind recognizes the following emotions: joy, distress, satisfaction, disappointment, gratitude, anger and shame. They are inferred from students' actions in the system interface based on an appraisal psychological model of emotion called $\mathrm{OCC}^{44}$. According to these models, the emotions are elicited by evaluating a situation, or a person's actions, based on his goals or standards, which is called appraisal ${ }^{45}$. In fact, Pat deduces the student's appraisal from his actions in the learning environment and his motivational orientation (intrinsic, extrinsic).

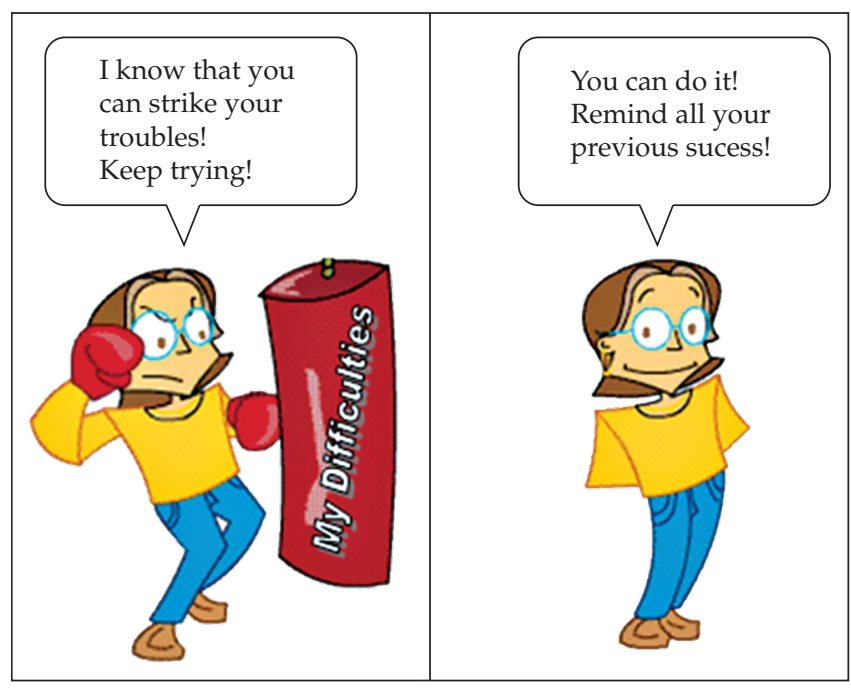

Figure 7. Examples of animations for "increase-student-self-ability" tactic.

Let us imagine a female student who has extrinsic motivational orientation and feels disappointed because she failed to accomplish a task correctly. She usually makes no additional effort when she faces difficulties and fails, because she feels unable to accomplish the task ${ }^{38}$, a frequent reaction by students who are extrinsic. In this situation Pat presents a message to enhance the student's beliefs about her self-ability and encourages her to carry out the task with a little more effort. The idea is to show the student who has a performance goal that not succeeding in a particular task does not imply in lack of ability.

For this situation, the Pat's Mind selects the following tactics, each one composed by a verbal behavior (VB) or a physical behavior (PB).

1) Increase-student-self-ability (VB: Increase-studentself-ability; PB: Encouragement);

2) Increase-student-effort (VB: Increase-student-effort; PB: Speak)

3) Offer-help (VB: Offers-help, PB: Speak).

For example, the first tactic is increase-student-self-ability, which means to promote in the student more positive beliefs about her self-ability. For this tactic, the Pat's Body shows both a verbal (VB) and a physical behaviors (PB). In the above example, for the increase student-self-ability tactic, the Body chooses arbitrarily a VB of type "student-self-ability" and a PB of type "Encouragement". Figure 7 exemplifies two different behaviors that can be chosen by the Body agent for the tactic "increase-student-self-ability".

All Pat behaviors were designed by a graphical designer and were defined with the help of an education psychologist. Further details about agent Pat and the choice of affective tactics can be found elsewhere ${ }^{34-36}$. 


\section{Available Characters}

The visual form and look of APAs play an important role in motivating and engaging users ${ }^{46}$. In order to define the character's appearance, we first developed a questionnaire with the aid from both a psychologist and a pedagogue. This questionnaire was answered by five primary school teachers, since we intend to embed the Body agent into an intelligent learning environment developed for 8-9 year-old children, called Civitas ${ }^{47}$. The questionnaire was composed by the following questions:

1. In a computer-based domain independent learning environment, which character is more adequate: a human or another? Justify.

2. Which should be the character's appearance for it to be appealing to adults and children without driving away their attention?

3. What kind of clothes should the character wear? Head, hair, arms, face, eyes, eyeglasses, etc...

4. Which should be the character's length in proportion to the screen length?

5. Should the entire body of the character appear? Justify.

6. Should it display facial reactions? Of what kind? In which moments? Justify.

7. Should be able to choose the colors of the character? If not, which colors do you think are more appropriate? Justify.

8. Should the character be static or should it move in the screen? Justify.

9. Should the user have the option of changing other properties rather than color in the character? Which ones? Why?

10. Which posture the character should exhibit at each moment? Which gestures? What kind of movement? Formal? Relaxed? Justify.

11. Should the character communicate by text, speech or both? Justify.

Answers to the questionnaire allowed us to define the following desirable characteristics:

- Characters should appear intellectual and happy;

- Characters should be of full-length and should have approximately $10 \mathrm{~cm}$;

- Students should have the option of choosing a character among several others;

- The system should allow students to hide/show the character.

We opted for designing various characters and allow the students accessing the environment to choose the one they like best. Previous studies showing that users prefer to interact with characters that match their own appearance and personality or ethnicity ${ }^{4,48,49}$ reinforced this option. In early experiments, we also verified that users prefer to choose the appearance of the character they interact with ${ }^{34}$.
Currently, three different characters created by a professional designer are available, illustrated in Figure 8: a boy, a female teacher, and a monkey. These characters were chosen due to the age group of students that will interact with the environment where the Body agent shall be inserted. The boy has the same age of students who will access Civitas ${ }^{47}$. The female represents their teacher (in general a young woman). And the monkey was chosen because children like to interact with pets that think and speak, as in a cartoon. Pat is a character defined in previous works $35,36,50,51$.

\section{Believability in Body Agent}

In order to appear more real to users, the APA must become believable, i.e., students should get involved with the agent in such a way that them practically believe it is for real $^{42}$.

According to Loyall and Bates ${ }^{52}$, the term 'believability' is employed in the sense of believable actors in dramatic art, meaning that the public or users forget their skepticism and feel that the character or agent is for real. Making an agent believable involves providing it with the aspects to express its personality. An agent that represents an interactive and believable animated character is called a Believable agent.

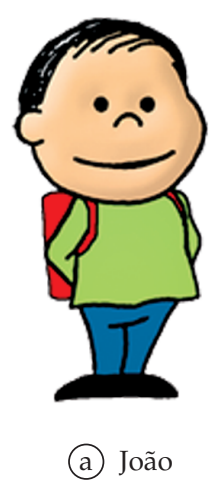

(C) Moly

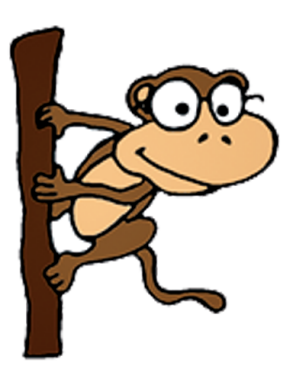

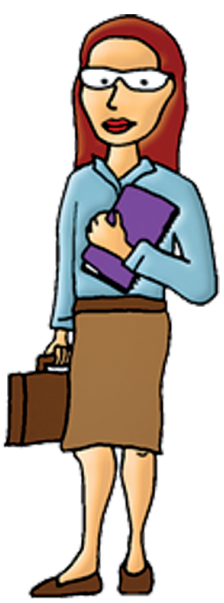

(b) Maria

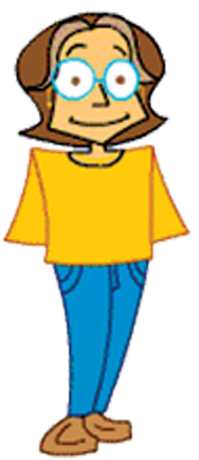

(d) Pat
Figure 8. Available Characters. 
Some actions can turn the agent more real, such as eye movements, a pause in speech, the conscience of body position and of personal space, and also communication in a natural language.

According to Hayes-Roth ${ }^{53}$, the animation behaviors must follow some premises for an animated agent to maintain credibility:

- There must be a varied repertoire of different behaviors to cover a great number of situations;

- There should be variability in the expression of a nominal type of behavior so that it looks alive and less robotic;

- The environment must not distract the user;

- There must be ambiguous behaviors that can be used in different contexts;

- There should be an attenuation of the behaviors so that observers experience a distribution of the expressive effect, requiring different levels of interpretation;

- There should be signature behaviors that occur with certain frequency in a context to designate the key character's qualities;

- The character must have particular attitudes that differentiate it from the others.

In order to be believable, the agent should show behaviors not directly related to pedagogical activities. For example, it may tap the foot on the ground or breathe when it is idle. To make our characters even more believable, we designed some physical behaviors for João, Maria and Moly. João can tap the foot on the ground, yawn or sit in a chair. Maria cleans her glasses or read her book. Moly makes some acrobatics and scratches his head. These idle behaviors are controlled by the Believable Behavior Manager, BBM (see Figure 5). When the Behavior Manager (BM) finishes showing a behavior, it activates the BBM that randomly displays the agent's idle behaviors every 3 minutes. BBM is also responsible for showing the idle behavior "blinks" every 5 seconds. When a tactic arrives, BM deactivates BBM and reactivates it again when the behavior tactic has been shown.

Nonetheless, we must always keep in mind that the goal of a pedagogical agent is to promote learning. Therefore, the agent's behavior must increase its believability, without reducing the learning effectiveness ${ }^{20}$. Any behavior that intervenes in the problem resolution by the student is inappropriate, regardless of how much it contributes to agent believability, e.g., an agent making acrobatics on the screen while a student is carrying out a difficult exercise would be certainly disturbing. For the agent to respect the criterion of controlled visual impact, aimed at keeping the student's attention in the pedagogical activity, we implemented a control mechanism for idle behaviors. When the student is accomplishing a task that requires concentration, the Mind agent sends a message to the Body agent to deactivate the exhibition of idle behaviors. In fact, the unique idle behavior still shown in this situation is "to blink". The Body agent activates idle behaviors once it receives a specific message of the
Mind agent or after 5 minutes. These time parameters may be configured in the agent Database Information Manager interface.

\section{Automatic Updating}

The possibility of including new characters and behaviors, as well as new tactics, requires a mechanism for installing new components and for automatic updates. The implemented mechanism is based on versions. When the system is started, the updating client module searches the server for new versions and updates it if necessary. As each version has the information on new features added (a new character, or a new behavior), the updating client does not need to reinstall the entire Body agent, but just download and copy the respective compacted file.

This mechanism consists of a Java class that connects to the server by sockets. It verifies if a new version is available and, if so, downloads and installs the corresponding files.

\section{Integration of the Body Module with other Learning Systems}

A learning system should meet certain restrictions in order to integrate the Body agent. First, the intelligent module of the educational system responsible for selecting affective tactics, the so-called Mind module, should communicate through FIPA-ACL in order to send the chosen tactics to the Body agent. If it uses different affective tactics from those already available in the Body agent, these should be registered into the system as explained in Section 5.2. If the learning system administrator desires to exhibit behaviors different than those already available in the Body agent database for these new tactics, they should be designed and inserted into the system database as explained in section 5.4, and also registered in the system. As previously mentioned, the system provides a friendly interface for registering new tactics and behaviors.

Two main problems may arise when the system is being executed. Network connection problems may interfere in the communication between the Mind and Body agents. Another problem is the Mind agent requesting a tactic that was not registered in the Body agent's database. In both cases, as the agent will not be able to show the behaviors related to the affective tactic, it will remain showing only believable behaviors (see Section 7).

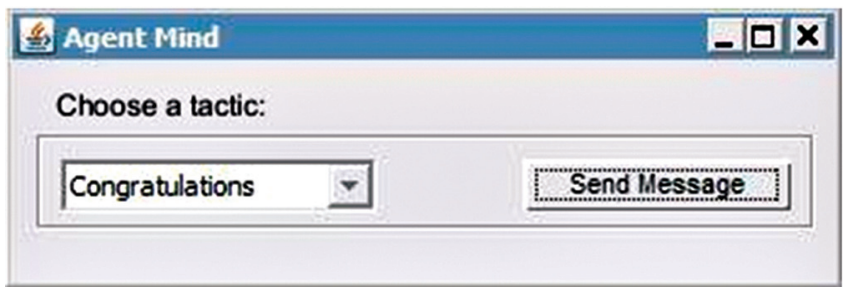

Figure 9. Interface for simulation of the Mind agent. 


\section{Conclusion and Future Work}

In order to test the Body agent, a simple environment that simulates the Mind agent was developed, as this work shall be used in the implementation of a lifelike interface agent. This environment allowed us to simulate the Mind agent sending a tactic to the Body agent and to study its behavior. Its interface is illustrated in Figure 9.

The Body agent is being employed to represent the character of a lifelike pedagogical agent whose goal is to assist students who have hearing impairments, communicating with them in sign language.

\section{Acknowledgements}

Many thanks to the graphical designer Jonatan Sarmento who created and designed the characters João, Maria and Moly.

We would like to thank CAPES for the support provided by resources from a CAPES/COFECUB project.

\section{References}

1. Lester J, Voerman JL, Towns SG and Callaway CB. Cosmo: a life-like animated pedagogical agent with deictic believability. In: Proceedings of IJCAI Workshop on Animated Pedagogical Agents: Making them Intelligent; 1997; Nagoya. San Francisco: Morgan Kaufmann; 1997. p. 61-69.

2. Paiva A and Machado I. Vincent, an autonomous pedagogical agent for on-the-job training. In: Proceedings of the 4 International Conference on Intelligent Tutoring Systems; 1998; San Antonio, Texas, USA. New York: Springer-Verlag; 1998. p. 584-593.

3. Burleson W and Picard RW. Gender-specific approaches to developing emotionally intelligent learning companions. IEEE Intelligent Systems 2007; 22(4):62-69.

4. Reeves B and Nass CI. The media equation: how people treat computers, television, and new media like real people and places. New York: Cambridge University Press; 1996.

5. Johnson WL, Shaw E and Ganeshan R. Pedagogical agents on the web. In: Proceedings of the ITS Workshop on Pedagogical Agents; 1998; San Antonio. p. 2-7.

6. Johnson WL, Shaw E, Marshall A and LaBore C. Evolution of user interaction: the case of agent adele. In: Proceedings of the 8 International Conference on Intelligent User Interfaces; 2003; Miami. New York: ACM; 2003. p. 93-100.

7. Rickel J and Johnson WL. Steve: a pedagogical agent for virtual reality. In: Proceedings of the 2 International Conference on Autonomous Agents; 1996; St. Paul, Minnepolis, USA. New York: ACM; 1996. p. 165-172.

8. Paiva A, Machado I and Martinho C. Enriching pedagogical agents with emotional behavior: the case of Vincent. In: Proceedings of the AIED Workshop on Animated and Personified Pedagogical Agents; 1999; Le Mans.

9. Reategui EB, Boff E, Ceron RF and Viccari RM. Um agente animado para ambientes de aprendizagem colaborativos. Revista Brasileira de Informática na Educação 2006; 14(3):27-38.
10. Rickel J and Johnson L. Animated pedagogical agents for team training. In: Proceedings of the ITS Workshop on Pedagogical Agents; 1998; San Antonio. p. 75-77.

11. André E, Rist T and Muller J. Employing AI methods to control the behavior of animated interface agents. Applied Artificial Intelligence 1999; 13(4):415-448.

12. Faivre J, Nkambou R and Frasson C. Integrating adaptive emotional agents in ITS. In: Proceedings of the 6 International Conference on Intelligent Tutoring Systems; 2002; Maceió, Brazil. Berlin: Springer-Verlag; 2002. p. 996-997.

13. Bica F, Verdin R and Vicari RM. Using the beliefs of self-efficacy to improve the effectiveness of ITS: an empirical study. In: Proceedings of Mexican International Conference on Artificial Intelligence; 2006; Apizaco, Mexico. Berlin: Springer-Verlag; 2006. p. 248-258.

14. Lester J, Converse S, Kahler S, Barlow S, Stone B and Bhogal R. The persona effect: affective impact of animated pedagogical agents. In: Proceedings of SIGCHI Conference on Human Factors in Computing Systems; 1997; Atlanta, Georgia. United States: ACM Press; 1997. p. 359-366.

15. Bercht $M$ and Viccari RM. Pedagogical agents with affective and cognitive dimensions. In: Proceedings do Congreso Iberoamericnao de Informatica Educativa; 2000; Santiago. Santiago: Universidad de Chile; 2000.

16. Jaques PA. Using an animated pedagogical agent to interact affectivelly with the student. [PhD thesis]. Porto Alegre: Universidade Federal do Rio Grande do Sul; 2004. p. 228.

17. Coles G. Reading lessons: the debate over Literacy. New York: Hill \& Wang; 1998.

18. Horstmann C. Big Java. Porto Alegre: Bookman; 2004.

19. Foundation for Intelligent Physical Agents - FIPA. FIPA Communicative Act Library Specification. Geneva, Switzerland; 2002.

20. Johnson WL, Rickel J and Lester J. Animated pedagogical agents: face-to-face interaction in interactive learning environments. International Journal of Artificial Intelligence in Education 2000; 11(1):47-78.

21. Picard RW. Affective computing. Cambridge: MIT Press; 1997.

22. Picard RW, Vyzas E and Healey J. Toward machine emotional intelligence: analysis of affective physiological state. IEEE Transactions Pattern Analysis and Machine Intelligence 2001; 23(10):1175-1191.

23. Wehrle $\mathrm{T}$ and Kaiser S. Emotion and facial expression. In: Paiva A. (Ed.). Affective interactions: towards a new generation of computer interfaces. Berlin: Springer; 2000. p. 49-63. (v. 1814)

24. Martinho C, Machado I and Paiva A. A cognitive approach to affective user modeling. In: Paiva A. (Ed.). Affective interaction. London: Springer; 2000. p. 64-75. (v. LNCS/1814)

25. Izard CE. Emotion cognition relationship and human development. In: Izard CE, Kagan J and Zajonc RB. (Eds.). Emotions, cognition and behavior. Oxford: Oxford University Press; 1984. p. 59-67.

26. Lester JC, Voerman J, Towns S and Callaway C. Deictic believability: coordinated gesture, locomotion and speech in 
lifelike pedagogical agents. Applied Artificial Intelligence 1999; 13(4-5):383-414.

27. Lester J and Towns S. Deicitic and emotive communication in animated pedagogical agents. In: Cassel J, Prevost S, Sullivan $\mathrm{J}$ and Churchill E. (Eds.). Embodied conversational agents. Cambridge: MIT Press; 2000. p. 123-154.

28. Elliott C. Affective reasoner personality models for automated tutoring systems. In: Proceedings of the 5 Workshop Pedagogical Agents; 1997; Kobe, Japan. p. 33-39.

29. AndréE, RistT and Müller JP. Life-like presentatin agent: a new perspective for computer-based technical documentation. In: Proceedings of the 5 Workshop Pedagogical Agents; 1997; Kobe, Japan. p. 1-8.

30. Abou-Jaoude S and Frasson C. Emotion computing in competitive learning environments. In: Proceedings of the 2 Workshop on Pedagogical Agents; 1998; San Antonio. p. 33-39.

31. Pelachaud C and Poggi I. Multimodal embodied agents. Knowledge Engineering Review 2002; 17(2):181-196.

32. Rehm M and André E. Catch me if you can: exploring lying agents in social settings. In: Proceedings of the 4 International Joint Conference on Autonomous Agents and Multiagent Systems; 2005; The Netherlands. New York: ACM; 2005. p. 937-944.

33. McQuiggan SW and Lester JC. Modeling and evaluating empathy in embodied companion agents. International Journal of Human Computer Studies 2007; 65(4): 348-360.

34. Jaques PA, Viccari RM, Pesty S and Bonneville JF. Applying Affective Tactics for a Better Learning. In: Proceedings of European Conference on Artificial Intelligence; 2004; Valencia, Spain. Amsterdam: IOS Press; 2004. p. 109-113.

35. Jaques PA, Lehmann M and Pesty S. Evaluating the affective tactics of an emotional pedagogical agent. In: Proceedings of the ACM Symposium on Applied Computing-Human Computer Interaction Track; 2009; Hawaii. New York: ACM; 2009. p. 104-109.

36. Jaques PA and Vicari RM. Infering emotions and applying affective tactics for a better learning. In: Vicari RM, Jaques PA and Verdin R. (Eds.). Agent-based tutoring systems by cognitive and affective modeling. New York: IGI Global; 2008. p. 135-155.

37. Brophy J. On motivating students. Michigan: Michigan State University; 1986.

38. Meece J and McColskey W. Improving Student motivation. Tallahassee, FL: Southeastern Regional Vision for Education (SERVE), 2001. Disponível em: <http://www.serve.org/ publications/rdism.htm>. Acesso em: Março 2002

39. Cameron J, Banko KM and Pierce WD. Pervasive negative effects on rewards on intrinsic motivation: the myth continues. The Behavior Analyst 2001; 24(1):1-44.
40. Bandura A. Self-efficacy. In: Ramachaudran R. (Ed.). Encyclopedia of Human Behavior. New York: Academic Press; 1994. p. 71-81.

41. Wilges B, Lucas JP and Silveira RA. Um agente pedagógico animadointegrado a um ambiente de ensino a distância. Renote Revista Novas Tecnologias na Educação 2004; 2(1):121-128.

42. BatesJ.Theroleofemotion in believableagents. Communication of ACM 1994; 37(7):122-125.

43. Chen P. The entity-relationship model: toward a unified view of data. ACM Transactions on Database Systems 1996; 1(1):9-36.

44. Ortony A, Clore G and Collins A. The cognitive structure of emotions. Cambridge: Cambridge University Press; 1988.

45. Scherer K. Appraisal theory. In: Dalgleish T and Power M. (Eds.). Handbook of cognition and emotion. New York: John Wiley \& Sons; 1999.

46. Gulz A and Haake M. Design of animated pedagogical agents: a look at their look. International Journal of Human-Computer Studies 2006; 64(4):322-339.

47. Axt M, Ferreira Filho RCM, Balle EE, Rodrigues SS and Muller DN. Cidades virtuais: tecnologias para aprendizagem e simulação. In: Anais do IV Seminário Jogos Eletrônicos, Educação e Comunicação; 2008; Salvador, Bahia. Salvador: UNEB; 2008. p. 1-10.

48. Baylor AL. Preliminary design guidelines for pedagogical agent interface image. In: Proceedings of the 10 International Conference on Intelligent User Interfaces; 2005; San Diego, California. New York: ACM; 2005; p. 249-250.

49. Baylor AL. Designing pedagogical agents to address diversity in learning. In: Proceedings of the 6 International Conference on Learning Sciences; 2004; Santa Monica, California. Mahwah: Lawrence Erlbaum Associates; 2004. p. 586-587.

50. Jaques PA, Jaques KSF and Lehmann M. Avaliando a efetividade de um agente pedagógico animado emocional. In: Anais do Simpósio Brasileiro de Informática na Educação; 2008; Fortaleza. Porto Alegre: SBC; 2008. CD-ROM.

51. Jaques PA. Avaliando um modelo afetivo de aluno baseado em uma abordagem cognitiva. In: Anais do Simpósio Brasileiro de Informática na Educação; 2008; Fortaleza. Porto Alegre: SBC; 2008. CD-ROM

52. Loyall AB and Bates J. Personality-rich believable agents that use language. In: Proceedings of the 1 International Conference on Autonomous Agents Marina del Rey; 1997; California. United States: ACM Press; 1997. p. 106-113.

53. Hayes-Roth B. Interacting with animated characters: puppets, bartenders and auto salespersons. Stanford: Knowledge Systems Laboratory; 1998. (Technical report) 
